\title{
Coordinate and synergistic effects of extensive treadmill exercise and ovariectomy on articular cartilage degeneration
}

\author{
Kazumasa Miyatake', Takeshi Muneta ${ }^{1}$, Miyoko Ojima', Jun Yamada', Yu Matsukura', Kahaer Abula',
} Ichiro Sekiya ${ }^{2}$ and Kunikazu Tsuji ${ }^{3 *}$

\begin{abstract}
Background: Although osteoarthritis $(\mathrm{OA})$ is a multifactorial disease, little has been reported regarding the cooperative interaction among these factors on cartilage metabolism. Here we examined the synergistic effect of ovariectomy (OVX) and excessive mechanical stress (forced running) on articular cartilage homeostasis in a mouse model resembling a human postmenopausal condition.

Methods: Mice were randomly divided into four groups, I: Sham, II: OVX, III: Sham and forced running (60 km in 6 weeks), and IV: OVX and forced running. Histological and immunohistochemical analyses were performed to evaluate the degeneration of articular cartilage and synovitis in the knee joint. Morphological changes of subchondral bone were analyzed by micro-CT.

Results: Micro-CT analyses showed significant loss of metaphyseal trabecular bone volume/tissue volume (BV/TV) after OVX as described previously. Forced running increased the trabecular BV/TV in all mice. In the epiphyseal region, no visible alteration in bone morphology or osteophyte formation was observed in any of the four groups. Histological analysis revealed that OVX or forced running respectively had subtle effects on cartilage degeneration. However, the combination of OVX and forced running synergistically enhanced synovitis and articular cartilage degeneration. Although morphological changes in chondrocytes were observed during $\mathrm{OA}$ initiation, no signs of bone marrow edema were observed in any of the four experimental groups.

Conclusion: We report the coordinate and synergistic effects of extensive treadmill exercise and ovariectomy on articular cartilage degeneration. Since no surgical procedure was performed on the knee joint directly in this model, this model is useful in addressing the molecular pathogenesis of naturally occurring OA.
\end{abstract}

Keywords: Ovariectomy, Mechanical stress, Forced running, Joint inflammation, Naturally occurring osteoarthritis model

\section{Background}

Osteoarthritis (OA) is a group of diseases and mechanical abnormalities involving degradation of articular cartilage and subchondral bone. Since OA is considered a multifactorial disease, the molecular pathogenesis of $\mathrm{OA}$ has proven difficult to fully elucidate [1]. Epidemiological analyses have identified several factors significantly associated

\footnotetext{
* Correspondence: tsuji.orj@tmd.ac.jp

${ }^{3}$ Department of Cartilage Regeneration, Tokyo Medical and Dental University, 1-5-45 Yushima, Bunkyo-ku, Tokyo 113-8519, Japan

Full list of author information is available at the end of the article
}

with OA prevalence. These include joint injury [2], joint instability (abnormal and/or excessive mechanical stress) $[3,4]$, joint inflammation $[5,6]$, menopause $[7,8]$, gender, aging, race $[9,10]$, and obesity $[11,12]$. Recent studies have revealed evidence that these risk factors may intricately and cooperatively contribute to both the initiation and progression of OA. Several prospective studies have confirmed that high body mass index (BMI) affects disease incidence more than it affects disease progression [13-15]. This may suggest that the combination of obesity and other mechanical factors, such as joint alignment and 
extensive stress, initiate OA. Other reports have suggested that obesity and estrogen have implications in the process of disease development as the association between high $\mathrm{BMI}$ and knee OA appears to be stronger in women compared with men [9-12]. Inflammation inside and in the surrounding region of the joint, including synovitis and bone marrow lesions (BML), is considered to be a major factor associated with the risk of both the progression and symptoms of OA such as joint pain [16-18]. The inflammation of the synovial membrane is reported to occur in both the early and late phases of OA and is associated with alterations in the adjacent cartilage tissues [19]. Synoviocytes are metabolically highly active cells residing in the synovial membrane and are considered physiologically prerequisite for joint homeostasis as they nourish chondrocytes via synovial fluid and remove metabolites and matrix degradation products from joint space. However, in an inflammatory environment, it is reported that these cells express catabolic and proinflammatory mediators such as cytokines, nitric oxide, prostaglandin E2, and neuropeptides, which leads to excess production of the proteolytic enzymes responsible for cartilage degeneration [16]. These evidences suggest the relevance of obesity, inflammation, and estrogen signal in disease pathogenesis.

Both prevention of cartilage loss and promotion of cartilage repair in the recovery of joint function are important issues to address for both the aged and young population. However, no etiotropic treatment for OA has been developed to date, and the current major therapeutic strategy for $\mathrm{OA}$ is based on conservative treatments such as muscle exercise with medication to relieve joint inflammation and pain [20]. One reason for this may be the complexity of the pathogenesis of OA as described above. To address the molecular pathogenesis of OA, several experimental OA models have been established using laboratory animals. These include surgical OA models involving conditions such as destabilization of the medial meniscus (DMM) [21], anterior cruciate ligament transection (ACL-T) [22], and menisectomy [3]. Other models use toxins or small chemical compounds which induce chondrocyte apoptosis or inflammation in the joint. Monosodium iodoacetate (MIA) or carrageenan are commonly used in these models [23, 24]. However, all these models seem to involve the pathogenesis of secondary (traumatic) OA, which represents less than $15 \%$ of total OA in humans [25].

This study was aimed to establish a new mouse OA model, which resembles the initiation and progression of human idiopathic (primary) OA. For this purpose, we examined the effect of extensive treadmill exercise (excessive mechanical stress) and ovariectomy (OVX) on articular cartilage homeostasis, both of which have been indicated as risk factors for human OA. We report both the coordinate and the synergistic effects of extensive treadmill exercise and ovariectomy on articular cartilage degeneration. Since no artificial procedure was performed on the knee joint directly in this model, we believe that this model is useful in addressing the molecular pathogenesis of idiopathic OA.

\section{Methods}

\section{Animal protocol}

Animal protocols were approved by the Institutional Animal Care and Use Committee (IACUC) of Tokyo Medical and Dental University (\#0140079A). Eight week-old female Balb/cCrSlc mice were randomly divided into two groups: the OVX group (OVX) and the control group (SHAM). Two weeks after surgery, all mice were subjected to forced running for 5 days at $12 \mathrm{~m} / \mathrm{min}$ for $10 \mathrm{~min}$ followed by $20 \mathrm{~m} / \mathrm{min}$ for $10 \mathrm{~min}$ using a rodent treadmill machine (Fig. 1a, MK-680R5; ME Service, Tokyo, Japan). Each group was further divided into two groups: one group was subjected to forced running by treadmill (OVX + Run or SHAM + Run) and the other group was left in the cage ad libitum (OVX + Cage or SHAM + Cage). The running group was forced to run $60 \mathrm{~km}$ for an additional 6 weeks (5 days per week) at $12 \mathrm{~m} / \mathrm{min}$ for $10 \mathrm{~min}$ of warm-up followed by $20 \mathrm{~m} / \mathrm{min}$ for $100 \mathrm{~min}$ (Fig. 1b). The number of mice in each experimental group is indicated in each figure. Mice were housed under a 12-h light-dark cycle and allowed food and water ad libitum. After 6 weeks, all the mice were sacrificed, and the uterus was extracted and weighed. Both left and right knee joints were also harvested for histological analyses. In addition, body weight was recorded before and after the running protocol. The humane endpoint was defined in this experiment according to the IACUC guidelines. If we observed indications of non-specific signs of illness such as inactivity, hunched posture or a rough coat during forced running by treadmill, experiments were terminated and mice were euthanized by carbon dioxide. Frequency of dropouts during treadmill running was about $30 \%$ in this experiment.

\section{Macroscopic observation of articular cartilage}

Right femurs were carefully dissected and articular cartilage was stained using India ink to visualize the damaged region as described by Sekiya et al. [4].

\section{Histology}

Left knee joints were harvested, and placed on a rubber plate at fixed knee joint angles $\left(30^{\circ}\right)$, fixed in $4 \%$ PFA (paraformaldehyde, SIGMA Aldrich, MO), demineralized in $20 \%$ EDTA (ethylenediaminetetraacetic acid) and embedded in paraffin. Five micrometer serial sagittal 


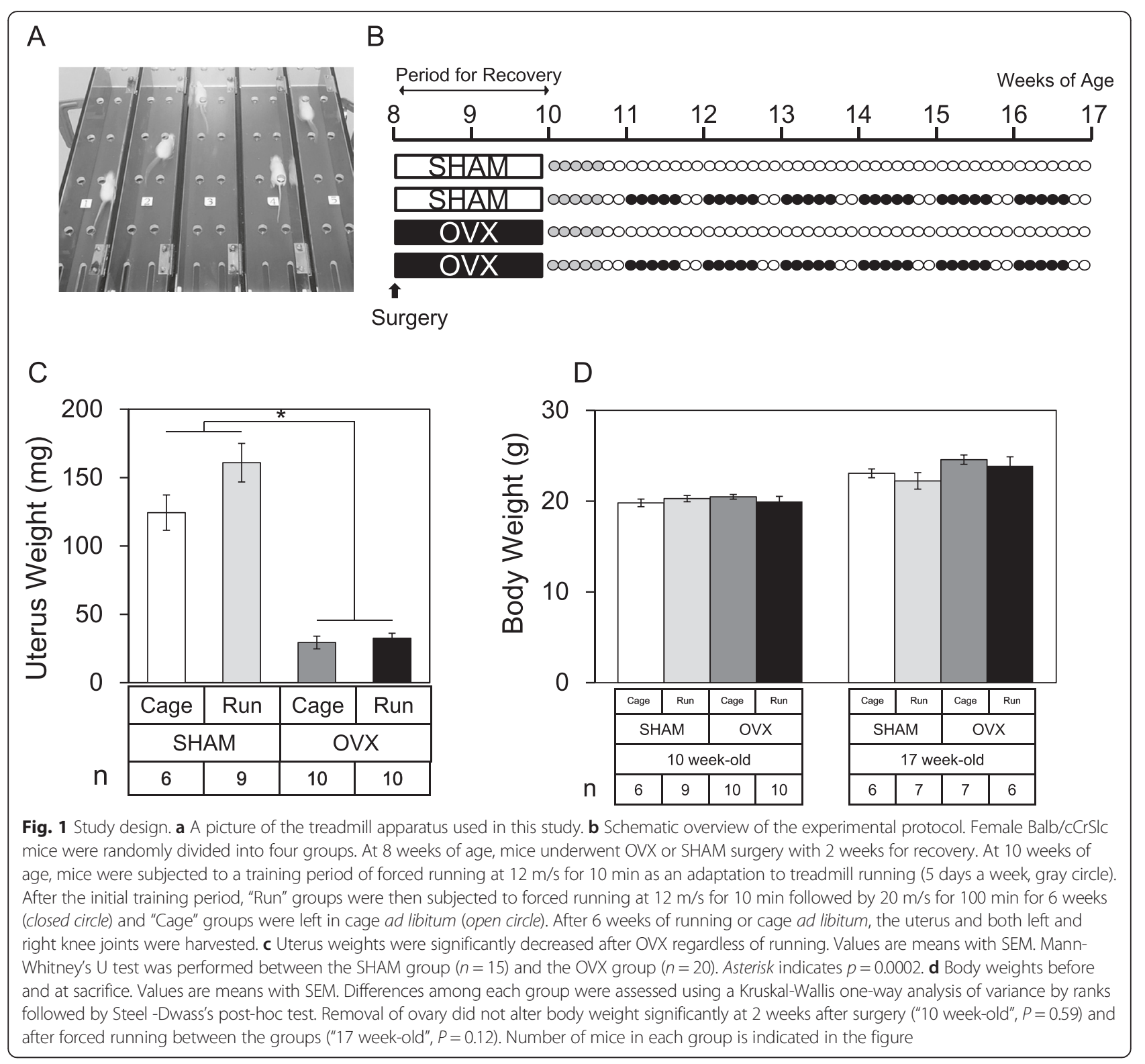

sections were prepared in the medial joint compartment, $150+/-20 \mu \mathrm{m}$ from the anterior cruciate ligament attachment site, for histological and immunohistochemical analyses. To evaluate cell infiltration and hyperplasia of the synovium, hematoxylin and eosin (H\&E) staining was performed. The severity of synovitis was evaluated as described by Yamada et al. (see below) [24]. To examine the loss of proteoglycan from articular cartilage, safranin-O staining was performed as we previously described [4]. The severity of articular cartilage damage was evaluated by the modified Mankin's score (see below) [2].

\section{Morphological analysis of articular cartilage}

Morphological parameters were evaluated as described by Abula et al. [26]. To evaluate chondrocyte cellularity, the size of each chondrocyte lacunae, and chondrocyte density in the articular cartilage, an area of articular cartilage was selected between the anterior and posterior edges of the meniscus. Carl Zeiss Axiovision software (Jena, Germany) was employed for the evaluation. Chondrocytes were counted and divided by the articular cartilage area to obtain chondrocyte density.

\section{Immunohistochemistry}

Paraffin-embedded sections were de-paraffinized in xylene, rehydrated through graded alcohol, and immersed in PBS. The samples were pretreated with $0.4 \mathrm{mg} / \mathrm{ml}$ proteinase $\mathrm{K}$ (DAKO, Carpinteria, CA, USA) in a Tris-HCl (pH 7.6) buffer for $15 \mathrm{~min}$ at room temperature for antigen retrieval. Any residual enzymatic activity was removed 
by washing with PBS, and nonspecific staining was blocked by pre-incubation with PBS containing $10 \%$ normal horse serum for $20 \mathrm{~min}$ at room temperature. Rabbit monoclonal anti-collagen type Ia1 and anti-collagen type IIa1 were purchased from Abcam (ab34710 and ab34712 Cambridge, MA, USA). Rat monoclonal anti-F4/80 was purchased from Bio-Rad (MCA497R Hercules, CA). Anti-collagen type I, type II collagen, and F4/80 (type I collagen 1:200 dilution; type II collagen 1:1000 dilution; F4/80 1:2000) were placed on each section for one hour at room temperature. After extensive washing with PBS, the sections were incubated in the secondary antibody of biotinylated anti-rabbit and rat IgG (Vector Laboratories, Burlingame, CA, USA) for $30 \mathrm{~min}$ at room temperature. Immunostaining was detected with VECTASTAIN ABC reagent (Vector Laboratories), followed by DAB staining. Counter staining was performed with Mayer's hematoxylin.

\section{Bone morphometric analysis (Micro computed tomography)}

To analyze the structural alteration of the knee joint after treadmill running and/or OVX, right femurs were harvested and fixed in $70 \%$ ethanol. Skeletal imaging was captured using a micro-CT apparatus (Scan XmateE090; Comscan Techno Co., Kanagawa, Japan). Both metaphyseal and epiphyseal bone volume per tissue volume (BV/TV), trabecular spacing (Tb. Spac.), trabecular thickness (Tb. Th.), trabecular number (Tb. N.), and subchondral bone plate thickness (Sb. Pl. Th.) of the tibia were calculated using Tri/3D-BONE software (Ratoc System Engineering Co., Tokyo, Japan). The trabecular bone parameter was measured in the metaphyseal region 300$800 \mu \mathrm{m}$ proximal from the edge of the growth plate.

\section{Modified Mankin's score}

Articular cartilage structure, tidemark duplication, safraninO staining, fibrocartilage, chondrocyte clones in uncalcified cartilage, and hypertrophic chondrocyte in calcified cartilage and subchondral bone were scored in both the femur and the tibia according to the modified Mankin's score (summed for a maximum score of 60) [2].

\section{Synovitis score}

Synovitis score was calculated as previously described by Yamada et al. [24]. Briefly, H\&E slides were used to evaluate synovial activation by scoring the thickening of the synovial lining and influx of inflammatory cells. In each knee joint, synovial activation was scored as follows: 0 , no changes compared to normal joints; 1 , thickening of the synovial lining and some influx of inflammatory cells; 2 , thickening of the synovial lining and intermediate influx of inflammatory cells; and 3, profound thickening of the synovial lining (more than four cell layers) and maximal observed influx of inflammatory cells. The upper and lower sides of the medial and lateral meniscus were scored and summed for a maximum score of 12 .

\section{Statistical analysis}

Statistical analysis was performed using Mann Whitney's U-test or Kruskal-Wallis one-way analysis of variance by ranks followed by Steel-Dwass's post-hoc methods. $P<$ 0.05 was considered as significant. Data is presented as the mean $+/$ - standard error of the mean (SEM).

\section{Results}

OVX and forced running synergistically accelerates articular cartilage degeneration in the knee joint

Weights of the uterus in the OVX group were significantly lower compared to those in the SHAM groups, indicating the complete removal of the ovary in the OVX group (Fig. 1c, $p=0.0002$ ). Since body weight is considered to be an important factor in articular cartilage homeostasis [12], we compared the average disparity of body weights between the groups during the experiment. As shown in Fig. 1d, regardless of OVX and forced running, we observed quite comparable body weights between the groups throughout the experimental period. In addition, macroscopic observation indicated the gross appearance of articular cartilage was quite comparable between the experimental groups. As shown in Fig. 2a, the cartilage surface remained smooth and we observed similar India ink dyeability regardless of OVX and forced running. However, we observed significant morphological changes in articular chondrocytes in the OVX group after forced running. As shown in Fig. 2b, ectopic hypertrophic chondrocytes were observed in the superficial zone of the tibia in the OVX + Run group (Fig. 2b arrow heads).

To further analyze articular cartilage morphology quantitatively, we measured cellularity (\% area of chondrocyte lacunae, Fig. 2c), size of chondrocyte $\left(\mu \mathrm{m}^{2}\right.$, Fig. 2d), and chondrocyte density (cells $/ 10^{4} \mu \mathrm{m}^{2}$, Fig. 2e) both beyond and below the tidemark of articular cartilage. As shown in Fig. 2c, cellularity in the superficial zones of articular cartilage was significantly increased in the OVX + Run group beyond the tidemark $(p=0.03)$. In the tibia, we found that the average size of each articular chondrocyte lacuna was increased beyond the tidemark in the OVX + Run group, although it was not statistically significant $(p=0.06)$. Quantitative analyses also indicated that the number of the cells was increased in the superficial layer of femur of the OVX + Run group (upper left panel, Fig. 2e, $p=0.06$ ) and in the deep layer of tibia (lower right panel, Fig. 2e, $p=0.04$ ).

To assess the effect of OVX and forced running on cartilage matrix degeneration, histological and immunohistochemical analyses were performed. As shown in Fig. 3a, OVX and forced running respectively had subtle 


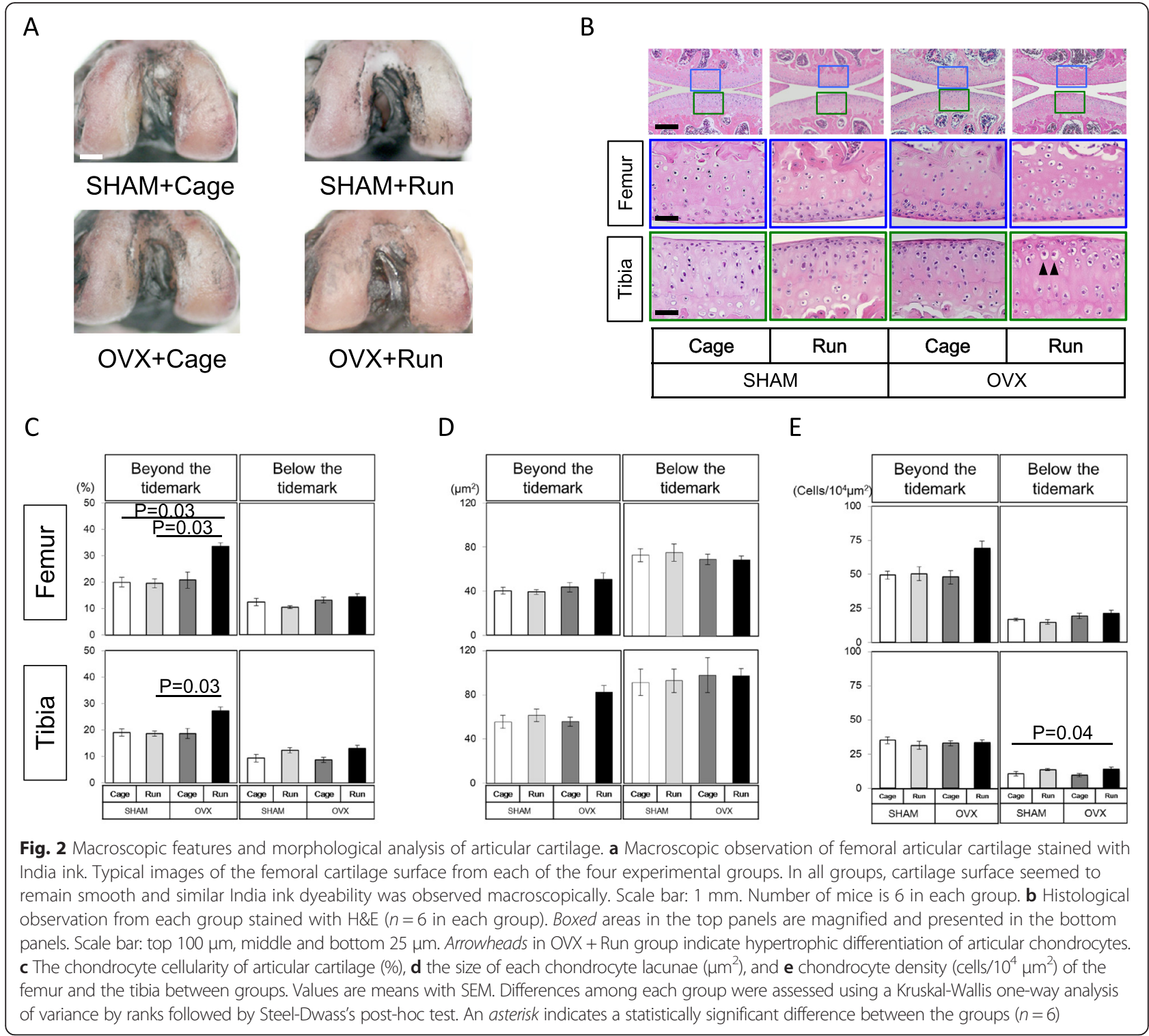

effects on safranin-O dyeability of articular cartilage. However, we observed significant loss of safranin-O dyeability in the OVX + Run group (Fig. 3a the rightmost column). To semi-quantitatively evaluate articular cartilage degeneration, the modified Mankin's score was calculated and plotted in Fig. 3b. The modified Mankin's score confirmed that OVX and forced running synergistically accelerated articular cartilage degeneration $(P$ $=0.04$ ). Immunohistochemical analyses indicated comparable levels of type II collagen expression in both femoral and tibial articular cartilage regardless of OVX and forced running (Fig. 3c). In contrast, we observed ectopic expression of type I collagen in both femoral and tibial articular cartilage in the OVX + Run group (Fig. 3d).
Effect of OVX and forced running on bone morphology Since abnormal subchondral bone metabolism such as osteoporosis, sclerosis, as well as osteophyte formation in the joint are thought to be associated with articular cartilage degeneration [27, 28], we performed bone morphometric analyses after OVX and forced running (Fig. 4). No apparent osteophyte formation or bone deformation around the knee joint was observed by micro-CT in any of the groups (Fig. 4a). Micro-CT analyses revealed that forced running increased both BV/TV and Tb. N. in the metaphyseal region of femur regardless of OVX, although these were not statistically significant (compare Cage and Run regardless of OVX, Fig. 4a and b). OVX reduced BV/ TV (compare SHAM and OVX regardless of running, Fig. 4a and b). In OVX mice, forced running for 6 weeks 


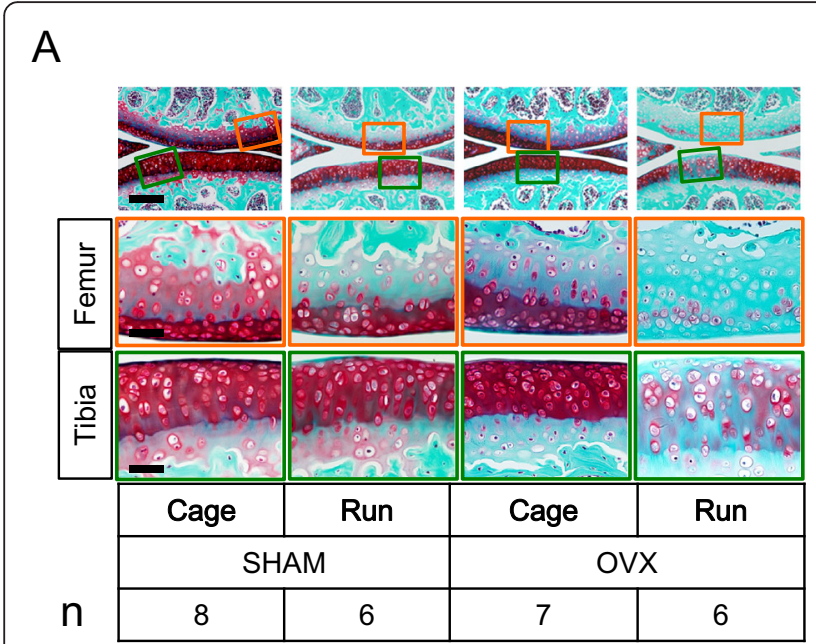

C

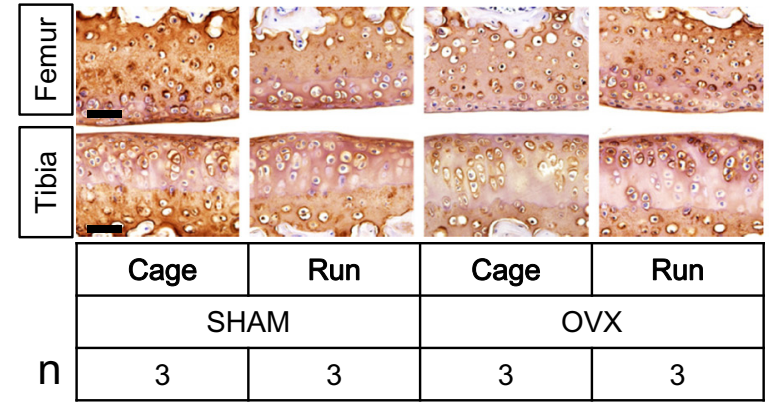

B

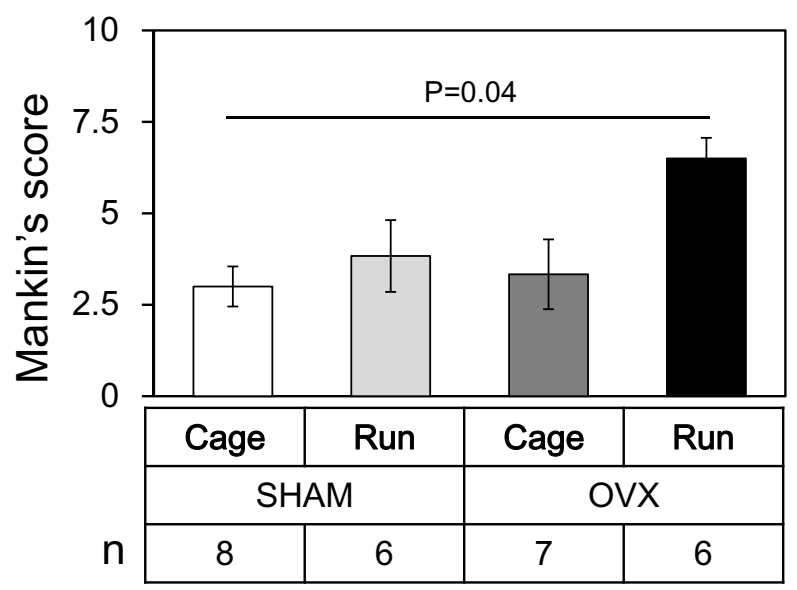

D

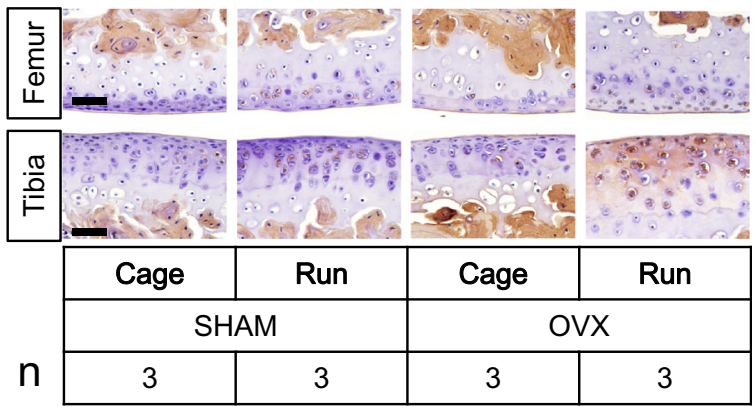

Fig. 3 OVX and forced running synergistically accelerates articular cartilage degeneration in the knee joint. a Histological observation from each group stained with safranin-O. Boxed areas in the top panels are magnified and presented in the bottom panels. We observed significant loss of safranin-O dyeability in the OVX + Run group. Scale bar: top $100 \mu \mathrm{m}$, middle and bottom $25 \mu \mathrm{m}$. Number of mice in each group is indicated in the figure. $\mathbf{b}$ Mankin's score for the femoral and tibial compartment between groups. The score confirmed that OVX and forced running synergistically accelerated articular cartilage degeneration. Values are means with SEM. Differences among groups were assessed by a Kruskal-Wallis one-way analysis of variance by ranks followed by Steel-Dwass's post-hoc test. Number of mice in each group is indicated in the figure. $\mathbf{c}$, $\mathbf{d}$ Immunohistochemistry for type II (c) and type I collagen (d) in each group. Ectopic expression of type I collagen in both femoral and tibial articular cartilage was observed in the OVX + Run group. Scale bar: $25 \mu \mathrm{m}$. Number of mice in each group is indicated in the figure

partially reversed BV/TV and Tb.N. up to the control level (SHAM + Cage) although this difference was not statistically significant (compare OVX + Cage and OVX + Run, Fig. 4b). In contrast, we did not observe any significant alteration in $\mathrm{Tb}$.Th. and $\mathrm{Tb}$. Spac. between the groups (Fig. 4b).

We observed distinct results in the metaphyseal (Fig. 4c) and epiphyseal (Fig. 4d) region of the tibia. BV/ TV was significantly increased after forced running in both metaphysis $(p=0.04)$ and epiphysis $(p=0.02)$ in control mice (compare SHAM + Cage and SHAM + Run in Fig. 4c, d). Interestingly, OVX abolished this effect (compare OVX + Cage and OVX + Run in Fig. 4c, d). In stark contrast to $\mathrm{BV} / \mathrm{TV}, \mathrm{Tb}$. N. was not increased in both metaphyseal and epiphyseal region of the tibia. Metaphyseal $\mathrm{Tb}$. Th. was significantly increased by forced running in sham mice (compare SHAM + Cage and SHAM + Run in Fig. 4c, $p=0.04)$. Similar to BV/TV, this effect was also deteriorated by OVX. We did not observe any significant alteration in Tb. Spac. between the groups (Fig. 4c and d). Thickness of the tibial subchondral bone plate was significantly increased by forced running (Fig. 4e, $p=0.01$ ), which was also deteriorated by OVX.

\section{OVX and forced running coordinately enhance joint inflammation}

Since inflammatory responses, such as inflammation in the synovial membrane and bone marrow edema in the epiphyseal bone marrow cavity were frequently observed at the onset of OA in the knee joint [16-18], we next probed for signs of joint inflammation in each group. In the SHAM + Run and OVX + Cage group we observed mild synovial hyperplasia and macrophage infiltration into the synovial membrane, both of which are indicators of joint inflammation (Fig. 5a). Interestingly, OVX 


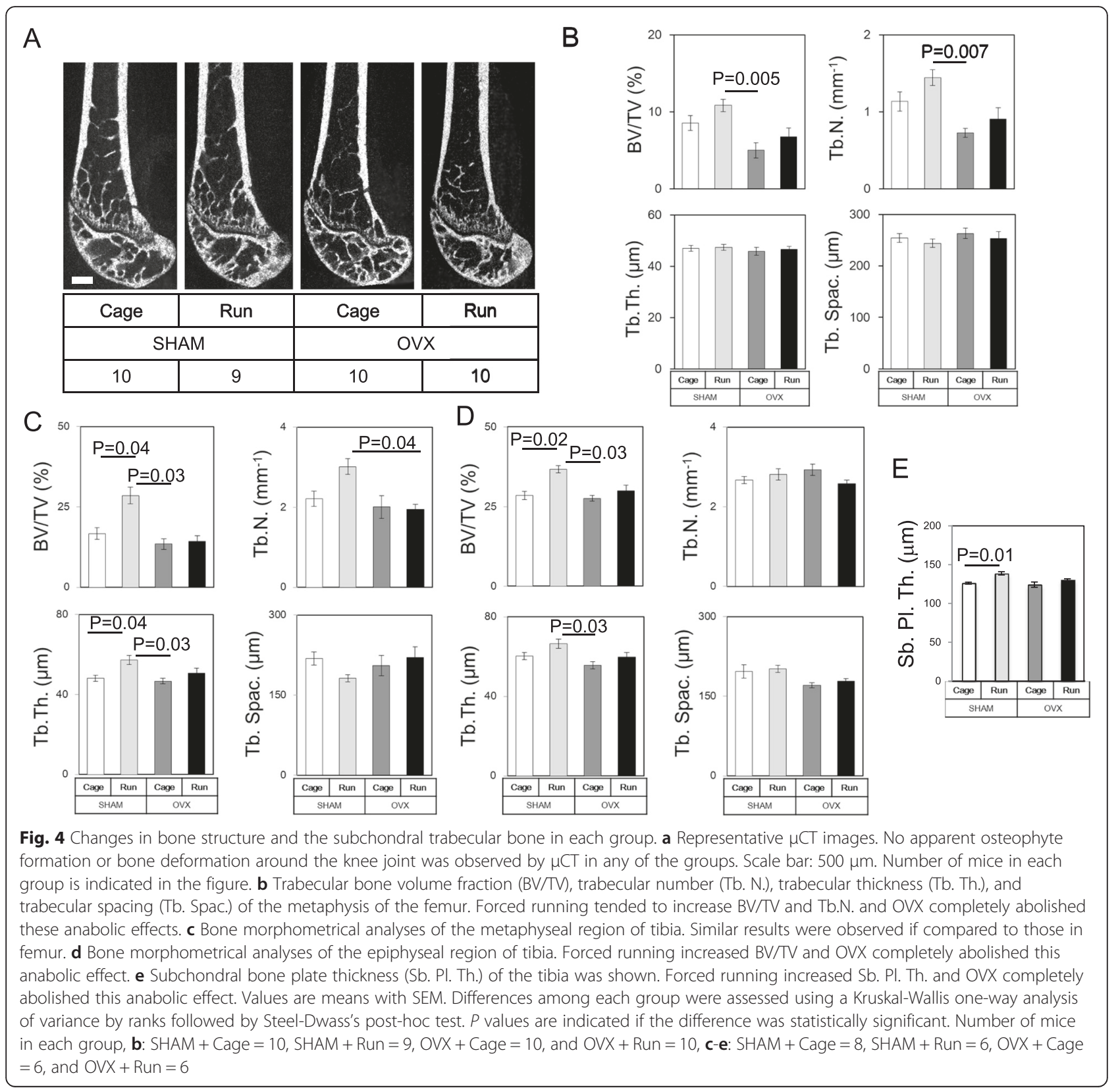

and forced running coordinately enhanced joint inflammation (Fig. 5a, OVX + Run, Fig. 5b). In contrast, we did not observe any significant change in cellularity or macrophage accumulation in the epiphyseal bone marrow cavity of the femur between the groups.

\section{Discussion}

In this study, we established a new mouse model which resembles naturally occurring OA phenotypes by combination of OVX and extensive mechanical stress to the knee joint. In this model, signs of early OA including ectopic type I collagen deposition, hypertrophic differentiation of articular chondrocytes, significant loss of proteoglycan, and synovitis were observed in the joint. We consider that this model is useful in addressing the molecular pathogenesis of naturally occurring $\mathrm{OA}$ since surgical procedure was not performed directly on the knee joint. Furthermore, this model will be useful to investigate the crosstalk between the signals activated post menopause and signals transduced by abnormal mechanical stress to the knee joint.

Menopause is a physiologic event characterized by loss of ovarian hormones and which can cause various disorders such as coronary heart disease, vasomotor symptoms, and osteoporosis [29]. In addition to mechanical stress such as joint instability [3] and extensive loading 
A

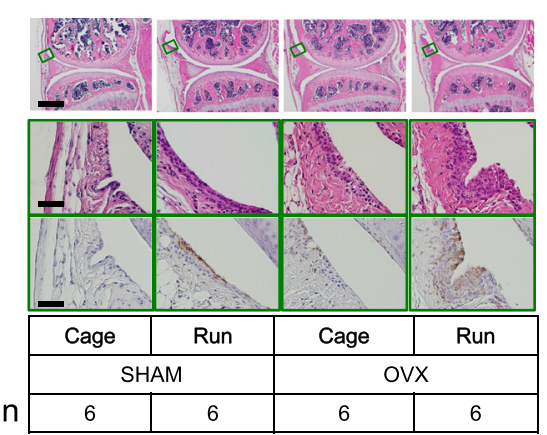

C

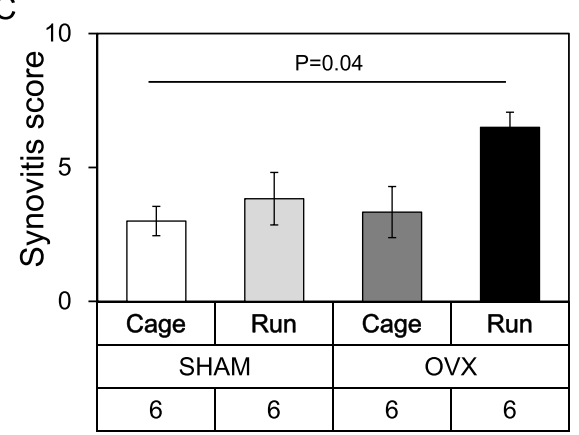

B

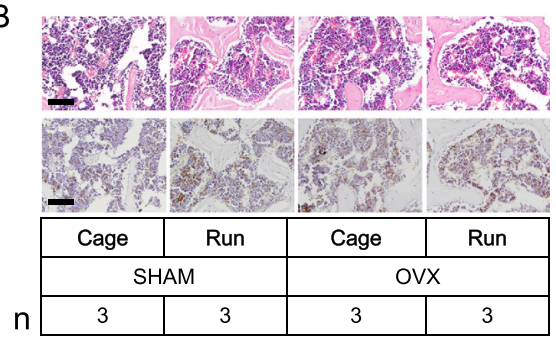

Fig. 5 Inflammatory response in each group. a H\&E staining and immunohistochemistry for F4/80 in the joint. Boxed areas in the top panels are magnified and presented in the bottom panels. Top and middle panels; H\&E staining, bottom panel; immunohistochemistry for F4/80 in the synovial membrane. Scale bar: top $250 \mu \mathrm{m}$, middle and bottom $25 \mu \mathrm{m}$. Number of mice in each group is indicated in the figure. $\mathbf{b}$ Histochemical analysis of epiphyseal bone marrow cavity of the femur. Scale bar: $25 \mu \mathrm{m}$. Top panels, H\&E staining. Bottom panels, F4/80 staining. Number of mice in each group is indicated in the figure. $\mathbf{c}$ Semi-quantitative analysis of synovial inflammation. Values are means with SEM ( $n=6)$. Differences among each group were assessed using a Kruskal-Wallis one-way analysis of variance by ranks followed by Steel-Dwass's post-hoc test. $P$ value is indicated if the difference is statistically significant

$[4,7,8,30,31]$, menopause is a major contributor to the degeneration of articular cartilage. In the current study, OVX induced significant bone loss. However, it did not induce significant effects on cartilage or synovium. Lugo et al. and Romen-Blas et al. reported that estrogen has both direct effects on cartilage and indirect effects on subchondral bone, muscle, ligament, and synovial membrane via estrogen receptor for joint homeostasis [32, 33]. Moreover, OVX is often accompanied by an increase in fat mass [34], which leads to increased levels of adipose tissue derived cytokines such as leptin and interleukin-6 (IL-6) [35, 36], which may also contribute to joint inflammation and OA progression. Sniekers et al. demonstrated that estrogen depletion increased the susceptibility of tissues in the joint for changes. However, an additional trigger induced by iodoacetate was needed to initiate osteoarthritic changes [37]. Taken together, our findings support the theory that additional triggers are required to initiate $\mathrm{OA}$ in postmenopausal women.

Exercise is widely recommended as one of the key preventive strategies for $\mathrm{OA}$ as well as osteoporosis. There are several animal studies reporting both the anabolic and catabolic effects of exercise on articular cartilage [4, 38-40], which suggest that moderate exercise is generally protective even after the onset of OA, although excessive exercise can lead to OA initiation and progression. Mechanical loading is reported to regulate the PTHrP/Ihh axis and presumed to be chondroprotective [41]. In our study, cartilage degeneration was always associated with excessive conditions, although the effect was not statistically significant when compared to control mice. This suggests that differences in age, strain, and species might also contribute to the susceptibility of OA initiation and progression.

Since OA is considered to be a degenerative joint disease characterized by cartilage breakdown as a consequence of wear and tear [42], the synovial membrane, and its inflammation have also been reported to play a physiologically important role on joint homeostasis and cartilage degradation as reported by Sellam et al. [16]. In addition, Blom et al. reported that synovial macrophages were crucial to cartilage degeneration in mediating Matrix metalloproteinase (MMP) activity in synovial macrophage knockout mice [43]. Several studies have indicated that inflammatory responses, such as bone marrow edema in the epiphyseal bone marrow cavity and inflammation in the synovial membrane, were frequently 
observed at the onset of OA in the knee joint. [16-18] As with previous reports, we observed the infiltration of macrophages into the synovial membrane in the OVX + RUN group, which might lead to the initiation of OA. Conversely, we found no alteration in bone marrow lesions between the 4 groups, although Roemer et al. reported the correlation between cartilage loss and bone marrow edema in a human study [17]. In addition, we found no apparent morphological alterations in deep zone cartilage despite significant morphological alterations in the superficial zone. Taken together, our results suggest that the synovium might account for OA initiation more than the bone marrow.

Type I collagen expression is believed to be a fibrocartilage marker [44]. Even though no decrease of type II collagen expression or alteration of the joint surface was observed macroscopically, fibrosis may have already occurred followed by an inflammatory response. In addition, our results also showed that the cellularity and hypertrophic chondrocytes were increased as well as ectopic type I collagen expression on articular cartilage in the OVX + Run group. Generally, hypertrophy of chondrocytes occurs during articular cartilage degeneration [3, 45]. Our data suggest that the combination of mechanical stress and the loss of ovary function enhanced the proliferation and hypertrophic differentiation of articular chondrocytes. Meanwhile, morphological changes in deep zone cartilage were not observed in any of the groups. One reason for this may be the fact that our current model represented a relatively early stage of OA compared to previous studies $[17,46]$. In addition, we believe that the increased chondrocyte cellularity, particularly in the upper cartilage zones, may be due to the fact that superficial cartilage becomes more exposed to factors from the synovium compared to deep zone cartilage.

Botter et al. reported that porosity in subchondral lesions due to increased osteoclastic activity was present in mouse OA induced by collagenaseVII [47]. As shown in Fig. 4, our study revealed that treadmill running always improved bone mineral parameters such as BV/TV and Tb.Th of both the femur and the tibia in accordance with previous human and animal studies $[48,49]$. However in most case, OVX completely abolished these anabolic effects. These data suggest the abnormal mechanotransduction in bone cells in the absence of estrogen signal. This may be one reason why we observed accelerated degradation in articular cartilage in OVX + Run group, further mechanistic analyses are required to elucidate this issue.

There are several limitations to the current study. First, although we reported the bone parameter of both the epiphysis and the metaphysis of the tibia, we could not examine the epiphysis of the femur according to the geometries of the epiphysis. However, the fact that the metaphysis bone parameter of the femur was similar to that of the tibia may suggest a similar correlation between the femur and the tibia on joint homeostasis. Second, the surgical approach was applied to young adult mice, and did not fully simulate the natural conditions of elderly postmenopausal women. Third, we focused on the synergistic effect of OVX and excessive mechanical stress, but we did not include estrogen supplementation in this study for the following reasons. Several studies have investigated the relationship between OVX and estrogen supplementation in young animals, lacking the premise that OVX is an established procedure which stimulates the hormonal condition of postmenopausal women; however, OVX not only changes estrogen levels, but also levels of progesterone (down), follicle-stimulating hormone (up), luteinizing hormone (up), and testosterone (down). Therefore, in surgical menopause models it is possible that estrogen supplementation might not compensate OVX. This may also explain why subsequent follow up analysis failed to show significant estrogen replacement therapy (ERT) protection against either the development or progression of radiographic knee OA in previous studies [50,51]. Fourth, we did not observe the long term combined effect of OVX and forced running. Since immunohistochemistry for type II collagen showed no apparent alteration in any of the four groups, a longer follow up period is necessary to assess any further alteration in type II collagen and its potential effect on OA change.

\section{Conclusions}

We established a new mouse model which resembles naturally occurring OA phenotypes by combination of OVX and extensive mechanical stress to the knee joint. We consider that this model is useful in addressing the molecular pathogenesis of naturally occurring OA since surgical procedure was not performed directly on the knee joint. Furthermore, this model will be useful to investigate the crosstalk between the signals activated post menopause and signals transduced by abnormal mechanical stress to the knee joint.

\section{Abbreviations \\ OA, osteoarthritis; OVX, ovariectomy; BV/TV, bone volume/tissue volume; Tb.Th., trabecular thickness; Tb.N., trabecular number; Tb.Spac., trabecular space (separation); Sb.PI.Th., subchondral bone plate thickness.}

\section{Acknowledgements}

All the authors thank Ms. Izumi Nakagawa for her administrative support to this study.

\section{Funding}

This study was supported by "the Project for Realization of Regenerative Medicine" from Japan Agency for Medical Research and Development (AMED) to IS and TM (1220000122011A182). This study was also supported by the grants from the Japan Society for the Promotion of Science (JSPS) to KT $(25293317,25460716,26462287,15$ K10464). 


\section{Availability of data and materials}

Data will be available upon request.

\section{Authors' contributions}

KM and KT made substantial contributions to the conception and design of this study, conducting the animal model studies, analysis and interpretation of data, and drafting the manuscript. JY, YM, and KA made contributions by conducting the animal model studies and the interpretation of data. $\mathrm{MO}$ made a contribution by conducting histology. IS, KT, and TM secured funding, participated in the design and coordination of the study, the interpretation of data and the drafting of the manuscript. All authors were involved in drafting the paper or revising it critically for important intellectual content and approved the submitted and final versions.

\section{Competing interests}

The authors declare that they have no competing interests.

\section{Consent for publication}

Not applicable.

\section{Ethics approval and consent to participate}

Animal protocols were approved by the Institutional Animal Care and Use Committee (IACUC) of Tokyo Medical and Dental University (Approval Number 0140079A).

\section{Author details}

${ }^{1}$ Department of Joint Surgery and Sports Medicine, Tokyo Medical and Dental University, Tokyo 113-8519, Japan. ${ }^{2}$ Center for Stem Cell and Regenerative Medicine, Tokyo Medical and Dental University, Tokyo 113-8519, Japan. ${ }^{3}$ Department of Cartilage Regeneration, Tokyo Medical and Dental University, 1-5-45 Yushima, Bunkyo-ku, Tokyo 113-8519, Japan.

\section{Received: 4 March 2016 Accepted: 24 May 2016}

\section{Published online: 31 May 2016}

\section{References}

1. Johnson VL, Hunter DJ. The epidemiology of osteoarthritis. Best Pract Res Clin Rheumatol. 2014;28:5-15.

2. Furman BD, Strand J, Hembree WC, et al. Joint degeneration following closed intraarticular fracture in the mouse knee: a model of posttraumatic arthritis. J Orthop Res. 2007;25:578-92.

3. Kamekura S, Hoshi K, Shimoaka T, et al. Osteoarthritis development in novel experimental mouse models induced by knee joint instability. Osteoarthritis Cartilage. 2005;13:632-41.

4. Sekiya I, Tang T, Hayashi M, et al. Periodic knee injections of BMP-7 delay cartilage degeneration induced by excessive running in rats. J Orthop Res. 2009;27:1088-92.

5. Pelletier JP, Martel-Pelletier J, Abramson SB. Osteoarthritis, an inflammatory disease: potential implication for the selection of new therapeutic targets. Arthritis Rheum. 2001:44:1237-47.

6. Goldring MB, Goldring SR. Osteoarthritis. J Cell Physiol. 2007;213:626-34.

7. Nevitt MC, Felson DT. Sex hormones and the risk of osteoarthritis in women: epidemiological evidence. Ann Rheum Dis. 1996;55:673-6.

8. Sniekers YH, Weinans H, Bierma-Zeinstra SM, et al. Animal models for osteoarthritis: the effect of ovariectomy and estrogen treatment - a systematic approach. Osteoarthritis Cartilage. 2008;16:533-41.

9. Yoshimura N, Muraki S, Oka H, et al. Prevalence of knee osteoarthritis, lumbar spondylosis, and osteoporosis in Japanese men and women: the research on osteoarthritis/osteoporosis against disability study. J Bone Miner Metab. 2009;27:620-8.

10. Dominick KL, Baker TA. Racial and ethnic differences in osteoarthritis: prevalence, outcomes, and medical care. Ethn Dis. 2004;14:558-66.

11. Jiang $L$, Tian $W$, Wang $Y$, et al. Body mass index and susceptibility to knee osteoarthritis: a systematic review and meta-analysis. Joint Bone Spine. 2012;79:291-7.

12. Lohmander LS, Gerhardsson de Verdier M, Rollof J, et al. Incidence of severe knee and hip osteoarthritis in relation to different measures of body mass: a population-based prospective cohort study. Ann Rheum Dis. 2009;68:490-6.

13. Wolfe F, Lane NE. The longterm outcome of osteoarthritis: rates and predictors of joint space narrowing in symptomatic patients with knee osteoarthritis. J Rheumatol. 2002;29:139-46.
14. Le Graverand MP, Brandt K, Mazzuca SA, et al. Progressive increase in body mass index is not associated with a progressive increase in joint space narrowing in obese women with osteoarthritis of the knee. Ann Rheum Dis. 2009;68:1734-8.

15. Yusuf E, Bijsterbosch J, Slagboom PE, et al. Association between several clinical and radiological determinants with long-term clinical progression and good prognosis of lower limb osteoarthritis. PLoS One. 2011;6:e25426.

16. Sellam J, Berenbaum F. The role of synovitis in pathophysiology and clinical symptoms of osteoarthritis. Nat Rev Rheumatol. 2010;6:625-35.

17. Roemer FW, Nevitt MC, Felson DT, et al. Predictive validity of within-grade scoring of longitudinal changes of MRI-based cartilage morphology and bone marrow lesion assessment in the tibio-femoral joint-the MOST study. Osteoarthritis Cartilage. 2012;20:1391-8.

18. Felson DT, Niu J, Guermazi A, et al. Correlation of the development of knee pain with enlarging bone marrow lesions on magnetic resonance imaging. Arthritis Rheum. 2007:56:2986-92.

19. Benito MJ, Veale DJ, FitzGerald O, et al. Synovial tissue inflammation in early and late osteoarthritis. Ann Rheum Dis. 2005;64:1263-7.

20. McAlindon TE, Bannuru RR, Sullivan MC, et al. OARSI guidelines for the non-surgical management of knee osteoarthritis. Osteoarthritis Cartilage. 2014:22:363-88.

21. Ma HL, Blanchet TJ, Peluso D, et al. Osteoarthritis severity is sex dependent in a surgical mouse model. Osteoarthritis Cartilage. 2007;15:695-700.

22. Williams JM, Felten DL, Peterson RG, O'Connor BL. Effects of surgically induced instability on rat knee articular cartilage. J Anat. 1982;134:103-9.

23. Guzman RE, Evans MG, Bove S, et al. Mono-iodoacetate-induced histologic changes in subchondral bone and articular cartilage of rat femorotibial joints: an animal model of osteoarthritis. Toxicol Pathol. 2003;31:619-24.

24. Yamada J, Tsuji K, Miyatake K, et al. Follistatin alleviates synovitis and articular cartilage degeneration induced by carrageenan. Int J Inflamm. 2014;2014:959271.

25. Brown TD, Johnston RC, Saltzman CL, et al. Posttraumatic osteoarthritis: a first estimate of incidence, prevalence, and burden of disease. J Orthop Trauma. 2006;20:739-44.

26. Abula K, Muneta T, Miyatake K, et al. Elimination of BMP7 from the developing limb mesenchyme leads to articular cartilage degeneration and synovial inflammation with increased age. FEBS Lett. 2015;589:1240-8.

27. Hayami T, Pickarski M, Zhuo Y, et al. Characterization of articular cartilage and subchondral bone changes in the rat anterior cruciate ligament transection and meniscectomized models of osteoarthritis. Bone. 2006;38:234-43

28. Im Gl, Kim MK. The relationship between osteoarthritis and osteoporosis. J Bone Miner Metab. 2014;32:101-9.

29. North American Menopause Society. Estrogen and progestogen use in postmenopausal women: 2010 position statement of The North American Menopause Society. Menopause. 2010;17:242-55.

30. Takasu N. Experimental study on the effect of forced running on occurrence of osteoarthritis in the knee of C 57 BL mice. Nihon Seikeigeka Gakkai Zasshi. 1992:66:1165-75.

31. Hyttinen M, Arokoski J, Parkkinen J, et al. Age matters: collagen birefringence of superficial articular cartilage is increased in young guineapigs but decreased in older animals after identical physiological type of joint loading. Osteoarthr Cartil. 2001;9:694-701.

32. Lugo L, Villalvilla A, Largo $R$, et al. Selective estrogen receptor modulators (SERMs): new alternatives for osteoarthritis? Maturitas. 2014;77:380-4.

33. Roman-Blas JA, Castañeda S, Largo R, Herrero-Beaumont G. Osteoarthritis associated with estrogen deficiency. Arthritis Res Ther. 2009;11:241.

34. Cavailles V. Estrogens and receptors: an evolving concept. Climacteric. 2002; 5 Suppl 2:20-6.

35. Simopoulou T, Malizos KN, lliopoulos D, et al. Differential expression of leptin and leptin's receptor isoform (Ob-Rb) mRNA between advanced and minimally affected osteoarthritic cartilage; effect on cartilage metabolism. Osteoarthritis Cartilage. 2007;15:872-83.

36. Ryu JH, Yang S, Shin Y, et al. Interleukin-6 plays an essential role in hypoxiainducible factor 2a-induced experimental osteoarthritic cartilage destruction in mice. Arthritis Rheum. 2011;63:2732-43.

37. Sniekers YH, Weinans $H$, van Osch GJ, van Leeuwen JP. Oestrogen is important for maintenance of cartilage and subchondral bone in a murine model of knee osteoarthritis. Arthritis Res Ther. 2010;12:R182.

38. Lapvetelainen T, Hyttinen M, Lindblom J, et al. More knee joint osteoarthritis $(\mathrm{OA})$ in mice after inactivation of one allele of type II procollagen gene but 
less $\mathrm{OA}$ after lifelong voluntary wheel running exercise. Osteoarthritis Cartilage. 2001;9:152-60.

39. Lapvetelainen T, Hyttinen MM, Saamanen AM, et al. Lifelong voluntary joint loading increases osteoarthritis in mice housing a deletion mutation in type Il procollagen gene, and slightly also in non-transgenic mice. Ann Rheum Dis. 2002;61:810-7.

40. Brisby H, Papadimitriou N, Runesson E, Sasaki N, Lindahl A, Henriksson HB. Moderate physical exercise results in increased cell activity in articular cartilage of the knee joint in rats. Cells Tissues Organs. 2013;198(3):237-48.

41. Chen X, Macica CM, Nasiri A, Broadus AE. Regulation of articular chondrocyte proliferation and differentiation by indian hedgehog and parathyroid hormone-related protein in mice. Arthritis Rheum. 2008;58(12):3788-97.

42. Felson DT. Clinical practice. Osteoarthritis of the knee. N Engl J Med. 2006; 354:841-8.

43. Blom $A B$, van Lent PL, Libregts $S$, et al. Crucial role of macrophages in matrix metalloproteinase-mediated cartilage destruction during experimental osteoarthritis: involvement of matrix metalloproteinase 3. Arthritis Rheum. 2007:56:147-57.

44. Li J, Gorski DJ, Anemaet W, et al. Hyaluronan injection in murine osteoarthritis prevents TGFbeta 1-induced synovial neovascularization and fibrosis and maintains articular cartilage integrity by a CD44-dependent mechanism. Arthritis Res Ther. 2012;14:R151.

45. Kawaguchi H. Endochondral ossification signals in cartilage degradation during osteoarthritis progression in experimental mouse models. Mol Cells. 2008;25:1-6.

46. Tessier JJ, Bowyer J, Brownrigg NJ, et al. Characterisation of the guinea pig model of osteoarthritis by in vivo three-dimensional magnetic resonance imaging. Osteoarthritis Cartilage. 2003;11:845-53.

47. Botter SM, van Osch GJ, Clockaerts S, et al. Osteoarthritis induction leads to early and temporal subchondral plate porosity in the tibial plateau of mice: an in vivo microfocal computed tomography study. Arthritis Rheum. 2011;63:2690-9.

48. Babatunde OO, Forsyth JJ, Gidlow CJ. A meta-analysis of brief high-impact exercises for enhancing bone health in premenopausal women. Osteoporos Int. 2012;23:109-19.

49. Iwamoto J, Takeda T, Ichimura S. Effect of exercise on tibial and lumbar vertebral bone mass in mature osteopenic rats: bone histomorphometry study. J Orthop Sci. 1998;3:257-63.

50. Hannan MT, Felson DT, Anderson JJ, et al. Estrogen use and radiographic osteoarthritis of the knee in women. The Framingham Osteoarthritis Study. Arthritis Rheum. 1990;33:525-32.

51. Spector TD, Nandra D, Hart DJ, Doyle DV. Is hormone replacement therapy protective for hand and knee osteoarthritis in women?: The Chingford Study. Ann Rheum Dis. 1997;56:432-4.

\section{Submit your next manuscript to BioMed Central and we will help you at every step:}

- We accept pre-submission inquiries

- Our selector tool helps you to find the most relevant journal

- We provide round the clock customer support

- Convenient online submission

- Thorough peer review

- Inclusion in PubMed and all major indexing services

- Maximum visibility for your research

Submit your manuscript at www.biomedcentral.com/submit 